\title{
Impact De L'évasion Des Malades Hospitalisés Sur La Mobilisation Des Recettes Financières A L'hôpital Général De Référence De Manono, En République Démocratique Du Congo
}

\author{
[Impact of the Escape of Hospitalized Illnesses on the Mobilization of Financial Revenues \\ at the General Reference Hospital of Manono, in the Democratic Republic of Congo] \\ Jean - Luc BANZE LUKANDA ${ }^{1}$, Héman KABEMBA BUKASA ${ }^{2 *}$, Sylvain KILIMA KUNDA ${ }^{3}$, \\ MUFITE KAPYA Danny ${ }^{4}$, NGOY SHINDANO Romain ${ }^{5}$ \\ ${ }^{1}$ Section de Gestion des Institutions de Santé, Institut Supérieur des Techniques Médicales de Manono, Manono, \\ République Démocratique Congo \\ ${ }^{2}$ Section des Sciences Infirmières, Institut Supérieur des Techniques Médicales de Lubao, Lubao, République \\ Démocratique Congo \\ ${ }^{3}$ Section de Gestion des Institutions de Santé, Institut Supérieur des Techniques Médicales de Kalemie, Kalemie, \\ République Démocratique Congo \\ ${ }^{4}$ Section des Sciences Infirmières, Institut Supérieur des Techniques Médicales de Manono, Manono, République \\ Démocratique Congo \\ ${ }^{5}$ Section de Gestion des Institutions de Santé, Institut Supérieur des Techniques Médicales de Lubumbashi, \\ Lubumbashi, République Démocratique Congo \\ *Auteur correspondant [Correspondingauthor]: Dr Héman KABEMBA BUKASA, Chef de Travaux;
}

\section{Résumé}

Introduction: L'évasion des malades en milieu hospitalier, surtout dans le contexte des pays en voie de développement, constitue un sérieux handicap dans le système d'autofinancement des ressources financières. Cette étude avait pour objectif de déterminer le niveau de déficit financier occasionné par l'évasion des malades, dans la mobilisation des recettes dans notre milieu.

Méthode : Il s'agissait d'une étude descriptive transversale et des techniques de revue documentaire et d'interview des personnels soignants et les gestionnaires, à l'hôpital général de référence de Manono, dans la province du Tanganyika (RDC). La période d'étude a été de deux ans, soit de Janvier 2019 à Décembre 2020.

Résultats : Sur 4864 malades hospitalisés durant la période d'étude, $301(6,2 \%)$ s'étaient évadés des différents services hospitaliers, surtout en médecine interne $(16,9 \% ; n=89)$, pédiatrie $(7,6 \% ; n=448)$ et chirurgie générale $(3,2 \% ; n=224)$. Les pertes financières causées par l'évasion des malades ont été de 6,5\%, soit 1858 USD sur 28650 USD. Ce déficit financier constitue une grande perte dans la quête de l'autonomie financière de l'hôpital général de référence de Manono.

Conclusion: Les efforts devraient être fournis pour réduire, voire empêcher, l'évasion des malades hospitalisés. Dans ce contexte de pauvreté, les priorités seraient la lutte contre les inégalités sociales, la concertation et 
l'implication des autorités politiques, administratives et coutumières dans la gestion commune de l'hôpital, et la mobilisation des populations au changement de mentalité. L'accent doit être mis sur les conséquences de l'évasion des malades sur la santé des soignés et le fonctionnement de l'hôpital.

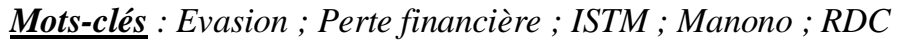

\begin{abstract}
Introduction: The escape of patients in hospitals, especially in the context of developing countries, constitutes a serious handicap in the system of self-financing of financial resources. The objective of this study was to determine the level of financial deficit caused by the escape of patients, in the mobilization of revenue in our community.

Method: This was a cross-sectional descriptive study supported by the techniques of documentary review and interviewing of nursing staff and managers, at the general reference hospital of Manono, in the province of Tanganyika (DRC). The study period was two years, from January 2019 to December 2020.

Results: Out of 4,864 patients hospitalized during the study period, 301 (6.2\%) had escaped from the various hospital services, especially in internal medicine $(16.9 \% ; n=89)$, pediatrics $(7.6 \% ; n=448)$ and general surgery (3.2\%; $n=224)$. The financial losses caused by the escape of the sick were $6.5 \%$, or $\$ 1,858$ out of $\$ 28,650$. This financial deficit constitutes a great loss in the quest for financial autonomy of the general reference hospital in Manono.
\end{abstract}

Conclusion: Efforts should be made to reduce or even prevent the escape of hospitalized patients. In this context of poverty, the priorities would be the fight against social inequalities, the consultation and involvement of political, administrative and customary authorities in the joint management of the hospital, and the mobilization of populations to change mentality. Emphasis must be placed on the consequences of the escape of the sick on the health of those treated and the functioning of the hospital.

Keywords: Evasion; Financial loss; ITSM; Manono; DRC

\title{
INTRODUCTION
}

L'évasion des malades constitue une barrièreindéniable au bon fonctionnement des structureshospitalières. Elle demeure d'une part un manque à gagner dans le système d'autofinancement des structures de soins envisagé par les gestionnaires ; d'autre part expose les malades évadés aux risques sanitaires liés à l'interruption des soins et de la confiance dans la relation malade- soignant.

En République Démocratique du Congo (RDC), la population active représente $40 \%$ et le milieu rural demeure encore plus peuplé [MANZAMBI et al.2015 ; MSP 2016]. Elle est classée parmi les pays pauvres et dont le système de santé dépend encore énormément de financement des bailleurs multilatéraux, bilatéraux et des organisations nongouvernementale (ONG) [MANZAMBI et al. 2015; MSP 2016] car 71,3\% de la population demeure dans la pauvreté alors que la croissance démographique se chiffre autour de 3,1\% [MSP 2016]. Selon le rapport sur les comptes de la santé RDC-2014, de 2010 à 2014 les dépenses totales moyennes de santé par habitant se chiffrent à 16,82 USD[MSP 2016]. Ce montant est de loin inférieur aux 44 USD préconisés par le «groupe de travail international sur le financement innovant des systèmes de santé » [MANZAMBI et al. 2015].

Le système de santé en RDC est organisé à trois niveaux : national, provincial ou régional (par les 26 divisions de la santé) et périphériques (zones de santé et ses aires de santé). Dans ce système, l'hôpital général de référence (HGR) est avant tout un milieu hospitalier qui accueille les malades et pratique des soins en rapport au paquet complémentaire d'activités (PCA). A cet effet, son fonctionnement harmonieux est garanti par des ressources adéquates à la fois humaines, matérielles et financières. En rapport à cette exigence, la barrière financière constitue 
DOI: $\underline{10.51386 / 25815946 / i j s m s-v 4 i 5 p 120}$

Volume: 4 Issue: 5

September to October 2021

https://www.ijsmsjournal.org

souvent un handicap pour accéder aux soins de qualité, surtout en milieu rural. D'où on peut assister à des pratiques telles que le recours à la médecine traditionnelle, la séquestration des malades par faute de payement de couts de soins, l'évasion des malades sans honorer la facture de soins, etc. [Chernichovsky et Meesook 1986 ; Larcher2007 ;Kabindaet al. 2019]

Les études spécifiques sur les évasions des malades en milieu hospitalier demeurent encore quasiment inexistantes dans la littérature médicale, surtout dans la région subsaharienne de l'Afrique. Cette étude semble être la première en RDC. Elle a été initiée dans l'objectif de déterminer le niveau de déficit financier, occasionné par l'évasion des malades hospitalisés, dans la mobilisation des recettes de l'hôpital général de référence de Manono.

\section{METHODES}

\section{Milieu d'étude}

Cette série d'étude a été menée à l'Hôpital Général de Reference de Manono, dans la Province du Tanganyika, au Sud-Est de la République Démocratique du Congo. Il s'agissait d'une structure Hospitalière ayant le paquet complémentaire d'activités (PCA), accueillant les malades du Territoire de Manono et ses environnants. C'est un hôpital rural, où exercent infirmiers et médecins généralistes. On y rencontre aucun spécialiste en médecine humaine. La population vit essentiellement de l'agriculture et de la pèche dans le fleuve Congo et ses affluents.

\section{Type Et Période d'étude}

Il s'agissait d'une étude descriptive transversale. Pour la récolte des données, nous avions utilisées les techniques de revue documentaire (cette technique nous a servi dans l'analyse de certains documents afin de pouvoir récolter les données fiables : les fiches des malades hospitalisés, les registres des malades, les registres des évadés, les rapports hebdomadaires de surveillance épidémiologique, les tableaux de bord des indicateurs de l'hôpital, les registres des recettes et le rapport de trésorerie) et d' 'interview (nous avions interviewé certains chefs des services médicaux, les Administrateurs Gestionnaires et les médecins pour recueillir les informations nécessaires sur les évasions des malades hospitalisés dans leur structure).

La période d'étude a été de deux ans, soit de Janvier 2019 à Décembres 2020.Durant cette période, les frais d'hospitalisation s'élevaient à 5000 francs congolais(2,5 - 2,6 USD) pour les enfants et à 10000 francs congolais (5 - 5,3 USD) pour les adultes. Ces frais inclus les actes médicaux et infirmiers posés sur le malade durant l'hospitalisation (injection, prise des signes vitaux, pose des voies veineuses, sondages urinaires, etc.) et l'occupation du lit pour une période de sept jours.

\section{Population d'étude et échantillon}

Notre population d'étude est constituée des malades hospitalisés à l'hôpital général de référence de de Manono. Les critères d'inclusion comprenaient tout malade hospitalisé durant la période d'étude avec dossiers complets, comprenant les paramètres recherchés.

Notre échantillon est systématique, de 4864 malades hospitalisés dans différents services hospitaliers, dont 2275 en 2019 et 2456 en 2020.

\section{Paramètres d'étude}

Les paramètres ci-après avaient été retenus parmi les malades évadés, enfin de réaliser cette étude et atteindre notre objectif : l'âge, le Sexe, la profession, l'Etat civil, le niveau d'instruction, le Service hospitalier du malade évadé, Frais d'hospitalisation non recouvrés par service. 


\section{DOI: $10.51386 / 25815946 /$ ijsms-v4i5p120}

Volume: 4 Issue: 5

September to October 2021

https://www.ijsmsjournal.org

\section{Analyse statistique des données}

Les données récoltées ont été encodés dans un fichier Excel et analysées (Microsoft 2016, USA).

\section{Définitions opérationnelles}

- Evasion de malade : Les malades évadés retenus étaient ceux qui devaient des dettes complètes ou incomplètes des soins à payer à la caisse de l'HGR Manono.

- «Motard » : Dans un sens local et pris dans cette étude, c'est le conducteur de Moto (motocycliste) qui fait payer ses services pour le transport en commun.

\section{RESULTATS}

Les résultats de cette série d'étude nous renseignent que 6,2\% (n=301) des malades sur un total de 4864 hospitalisés s'étaientévadés (clandestinement) de l'HGR de Manono de 2019 à 2020. Ces évasions ou fuites clandestines des maladeshospitalisés avaient représenté une perte de 1858 USD (soit 6,5\%) dans la mobilisation des recettes des hospitalisations des malades ( $\mathrm{n}=28650$ USD). La majorité des évasions des malades hospitalisés a été observée en $2019(3,5 \% ; n=168)$ contre $2,7 \%(n=133)$ en 2020, surtout de Juillet à Janvier.

Tableau 1. Répartitionsociodémographique des malades hospitalisés[Sociodemographic distribution of hospitalized patients]

\begin{tabular}{|c|c|c|c|c|c|c|}
\hline \multirow[t]{2}{*}{ Paramètres } & \multicolumn{2}{|c|}{ Total } & \multicolumn{2}{|c|}{ Malades évadés } & \multicolumn{2}{|c|}{ Malades non évadés } \\
\hline & $\mathbf{n}$ & $\%$ & $\mathbf{n}$ & $\%$ & $\mathbf{n}$ & $\%$ \\
\hline \multicolumn{7}{|l|}{ Age } \\
\hline 0 à 15 ans & 2177 & 44,8 & 166 & 7,6 & 2011 & 92,4 \\
\hline 16 ans et plus & 2687 & 55,2 & 135 & 5,0 & 2552 & 95,0 \\
\hline Total & 4864 & 100,0 & 301 & 6,2 & 4563 & 93,8 \\
\hline Moyen & 27,4 & & & & & \\
\hline $\mathrm{SD}$ & 1,3 & & & & & \\
\hline \multicolumn{7}{|l|}{ Sexe } \\
\hline Masculin & 1990 & 40,9 & 149 & 7,5 & 1841 & 92,5 \\
\hline Féminin & 2874 & 59,1 & 152 & 5,3 & 2722 & 94,7 \\
\hline \multicolumn{7}{|l|}{ Niveau d'instruction } \\
\hline Instruit & 2725 & 56,0 & 88 & 3,2 & 2637 & 96,8 \\
\hline Non instruit & 2139 & 44,0 & 213 & 10,0 & 1926 & 90,0 \\
\hline \multicolumn{7}{|l|}{ Profession } \\
\hline Cultivateur & 1643 & 33,8 & 151 & 9,2 & 1492 & 90,8 \\
\hline Sans emploi & 1667 & 34,3 & 121 & 7,3 & 1546 & 92,7 \\
\hline Enseignant & 653 & 13,4 & 4 & 0,6 & 649 & 99,4 \\
\hline Elève & 634 & 13,0 & 16 & 2,5 & 618 & 97,5 \\
\hline Motard* & 267 & 5,5 & 9 & 3,4 & 258 & 96,6 \\
\hline \multicolumn{7}{|l|}{ Etat civil } \\
\hline Marié & 2289 & 47,1 & 107 & 4,7 & 2182 & 95,3 \\
\hline Veuf (ve) & 21 & 0,4 & 8 & 38,1 & 13 & 61,9 \\
\hline Célibataire & 2554 & 52,5 & 186 & 7,3 & 2368 & 92,7 \\
\hline
\end{tabular}

*Se référer aux définitions opérationnelles [Refer to operationaldefinitions] 


\section{DOI: $10.51386 / 25815946 /$ ijsms-v4i5p120}

Volume: 4 Issue: 5

September to October 2021

https://www.ijsmsjournal.org

L'âge moyen des malades hospitalisés a été de 27,4 $\pm 1,3$ ans. La grande proportion des évadés concernait la tranche d'âge $\leq 15$ ans $(7,6 \% ; n=166)$ Vs. 5,0\% $(n=135)$ pour les malades dont l'âge a été $\geq 16$ ans ; de sexe masculin $(7,5 \% ; n=149)$ Vs. le sexe féminin $(5,3 \% ; n=152)$ avec un sex ratio de 1,$4 ;$ les malades sans instruction $(10,0 \%$; $\mathrm{n}=213)$ Vs. les malades instruits $(3,2 \% ; \mathrm{n}=88)$.

Tableau 2. Répartition des malades hospitalisés selon les services [Distribution of hospitalized patients by clinical services]

\begin{tabular}{|c|c|c|c|c|c|c|}
\hline \multirow[t]{2}{*}{ Service } & \multicolumn{2}{|c|}{ Total } & \multicolumn{2}{|c|}{ Malades évadés } & \multicolumn{2}{|c|}{ Malades non évadés } \\
\hline & $\mathbf{n}$ & $\%$ & $\mathbf{n}$ & $\%$ & $\mathbf{n}$ & $\%$ \\
\hline Médecine interne homme & 261 & 5,4 & 40 & 15,3 & 221 & 84,7 \\
\hline Médecine interne femme & 267 & 5,5 & 49 & 18,4 & 218 & 81,6 \\
\hline Sous -total (MI) & 528 & 10,9 & 89 & 16,9 & 439 & 83,1 \\
\hline Gynécologie et obstétrique & 1068 & 22,0 & 11 & 1,0 & 1057 & 99,0 \\
\hline Chirurgie générale & 1091 & 22,4 & 35 & 3,2 & 1056 & 96,8 \\
\hline Pédiatrie & 2177 & 44,8 & 166 & 7,6 & 2011 & 92,4 \\
\hline
\end{tabular}

Tableau 3. Répartition des malades hospitalisés selon les années d'étude[Distribution of hospitalized patients by year of study]

\begin{tabular}{|c|c|c|c|c|c|c|c|c|c|c|}
\hline \multirow[t]{3}{*}{ Mois } & \multirow{2}{*}{\multicolumn{2}{|c|}{ Total }} & \multicolumn{4}{|c|}{2019} & \multicolumn{4}{|c|}{2020} \\
\hline & & & Mala & vadés & $\begin{array}{l}\text { Malad } \\
\text { évadé }\end{array}$ & & $\begin{array}{l}\text { Malac } \\
\text { évadé }\end{array}$ & & Malade & évadés \\
\hline & $\mathbf{n}$ & $\%$ & n & $\%$ & n & $\%$ & $\mathbf{n}$ & $\%$ & n & $\%$ \\
\hline Janvier & 498 & 10,2 & 24 & 4,8 & 221 & 44,4 & 7 & 1,4 & 246 & 49,4 \\
\hline Février & 474 & 9,7 & 19 & 4,0 & 216 & 45,6 & 3 & 0,6 & 236 & 49,8 \\
\hline Mars & 435 & 8,9 & 11 & 2,5 & 219 & 50,3 & 3 & 0,7 & 202 & 46,4 \\
\hline Avril & 496 & 10,2 & 6 & 1,2 & 235 & 47,4 & 2 & 0,4 & 253 & 51,0 \\
\hline Mai & 415 & 8,5 & 16 & 3,9 & 197 & 47,5 & 2 & 0,5 & 200 & 48,2 \\
\hline Juin & 348 & 7,2 & 11 & 3,2 & 116 & 33,3 & 8 & 2,3 & 213 & 61,2 \\
\hline Juillet & 344 & 7,1 & 8 & 2,3 & 150 & 43,6 & 9 & 2,6 & 177 & 51,5 \\
\hline Aout & 365 & 7,5 & 16 & 4,4 & 137 & 37,5 & 8 & 2,2 & 204 & 55,9 \\
\hline Septembre & 336 & 6,9 & 9 & 2,7 & 148 & 44,0 & 20 & 6,0 & 159 & 47,3 \\
\hline Octobre & 454 & 9,3 & 17 & 3,7 & 201 & 44,3 & 28 & 6,2 & 208 & 45,8 \\
\hline Novembre & 353 & 7,3 & 10 & 2,8 & 157 & 44,5 & 27 & 7,6 & 159 & 45,0 \\
\hline Décembre & 346 & 7,1 & 21 & 6,1 & 110 & 31,8 & 16 & 4,6 & 199 & 57,5 \\
\hline Total & 4864 & 100,0 & 168 & 3,5 & 2107 & 43,3 & 133 & 2,7 & 2456 & 50,5 \\
\hline
\end{tabular}

En ce qui concerne la profession, les cultivateurs $(9,2 \% ; n=151)$, les sans emploi $(7,3 \% ; n=121)$ et les motards $(3,4 \% ; n=9)$ ont été répertoriés comme les plus grands groupes avec forte probabilité d'évasion suite à leur proportion. Les informations concernant l'état matrimonial (état civil) nous renseignent que les mariés constituent le groupe avec moins d'évasions des malades en hospitalisation $(4,7 \% ; n=107)$. 


\section{DOI: $10.51386 / 25815946 /$ ijsms-v4i5p120}

Volume: 4 Issue: 5

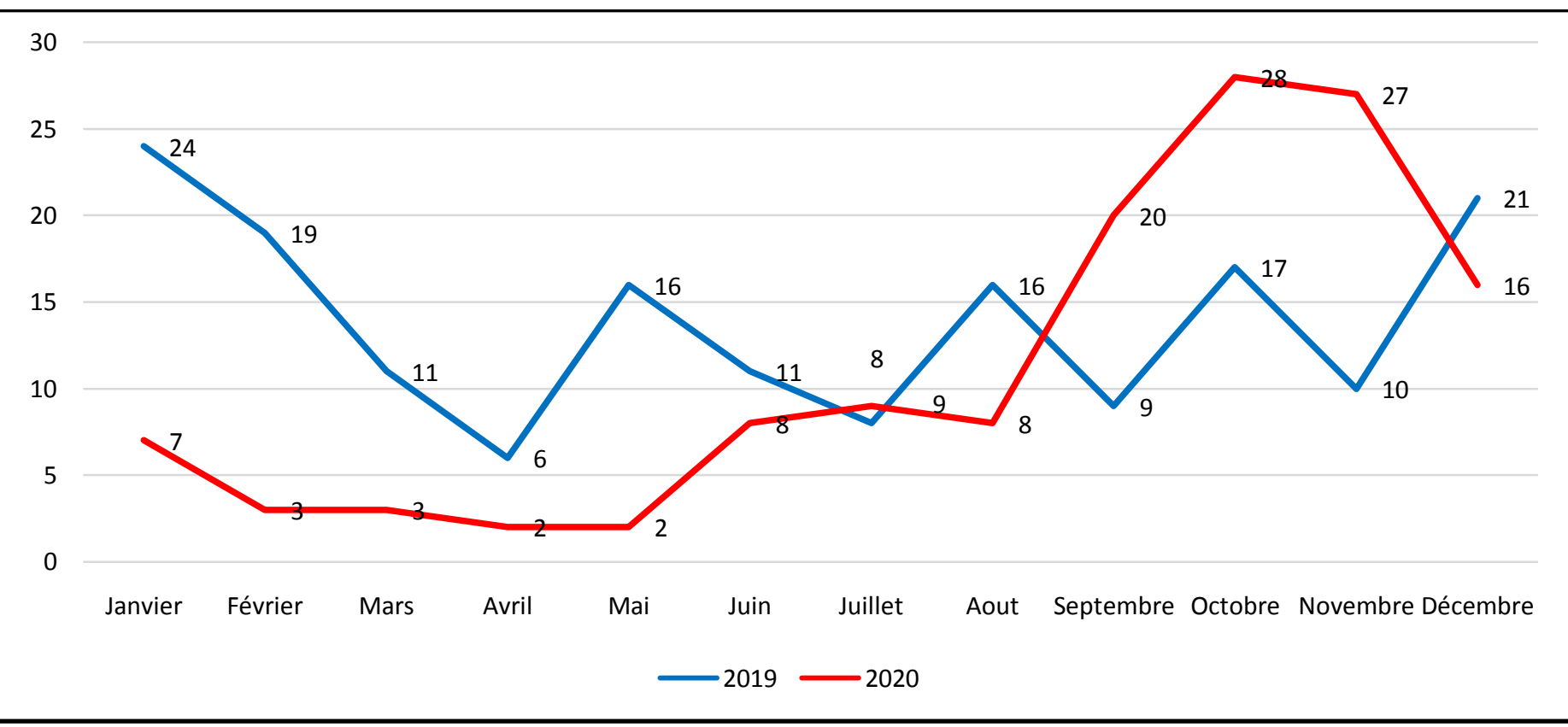

Figure 1. Évolution des évasions des malades à l'hôpital général de référence de Manono selon les Mois (2019 - 2020) [Evolution of patient escapes at Manono General Reference Hospital by Month (2019 - 2020)]

Tableau 4. Répartition des malades selon les entrées financières par service hospitalier [Distribution of patients according to financialreceipts by hospital service]

\begin{tabular}{lcccccc}
\hline Service & Total* & \multicolumn{3}{c}{ Malades évadés } & \multicolumn{3}{c}{ Malades non évadés } \\
& n & \% & n & \% & \multicolumn{1}{c}{ n } & \% \\
\hline Médecine interne homme & 1529 & 4,8 & 250 & 16,4 & 1278 & 83,6 \\
Médecine interne femme & 1568 & 4,9 & 204 & 13,0 & 1364 & 87,0 \\
Sous -total (MI) & 3097 & 9,8 & 454 & 14,7 & 2642 & 85,3 \\
Gynécologie et obstétrique & 3057 & 9,6 & 130 & 4,3 & 2927 & 95,7 \\
Chirurgie générale & 15615 & 49,2 & 739 & 4,7 & 14876 & 95,3 \\
Pédiatrie & 6881 & 21,7 & 535 & 7,8 & 6346 & 92,2 \\
Total & 28650 & 100,0 & 1858 & 6,5 & 26791 & 93,5 \\
\hline
\end{tabular}

*Montanten USD payéen CDF au taux de 1900 CDF (2019) et 2000 CDF (2020) [Amount in USD paid in CDF at the rate of 1900 CDF (2019) and 2000 CDF (2020)]

Les services de Chirurgie générale (49,2\%; $n=15615$ USD), pédiatrie $(21,7 \%$; $=6681$ USD) et médecine interne (9,8\%; $n=3097$ USD), suivi de gynécologie et obstétrique (y compris la maternité) avec 9,6\% ( $\mathrm{n}=3057$ USD) constituent les principales sources des recettes financières de l'HGR de Manono en ce qui concerne les hospitalisations des malades. Par contre (inverse), les services de médecine interne, pédiatrie et chirurgie générale constituent les services avec grandes évasions des malades, soit respectivement 16,9\% $(n=89), 7,6 \%(n=448)$ et $3,2 \%(n=224)$. 
DOI: $\underline{10.51386 / 25815946 / i j s m s-v 4 i 5 p 120}$

Volume: 4 Issue: 5

September to October 2021

https://www.ijsmsjournal.org

\section{DISCUSSION}

La problématique des évasions des malades est souvent abordée dans la littérature médiatique (journalistique) que scientifique. A cet effet, il existe toujours une difficultéà réaliser une discussion de qualité lorsqu'on aborde les thématiques des études moins menées par des chercheurs en santé publique et ses branches corollaires. C'est le cas de cette étude, qui semble être la première à être entreprise et publiée dans notre pays et région subsaharienne.

Durant la période de notre enquête, 6,2\% des malades hospitalisés s'étaient évadés de l'HGR de Manono.La majorité des évasions s'observent de Juillet à janvier, qui sont des périodes de soudures entre deux récoltes pour les agriculteurs de la région. Cette proportion est élevée au regard des risques auxquels les auteurs des évasions sont exposés car normalement la sortie du milieu hospitalier est conditionnée par le bilan clinique et paraclinique qui témoignent du rétablissement de la santé du malade. Toutefois, il est le reflet du niveau de pauvreté et du niveau de satisfaction des besoins de santé, avec les inégalités sociales comme dénominateur commun [Larcher 2007; Connolly et al. 2017;Kabindaet al. 2019]. Un proverbe populaire souligne bien cette réalité, où le manque d'un bien ou service peut entrainer la surdité et opposition : «Ventre affamé n'a point d'oreilles ». C'est pour cette raison que nous partageons les avis des Kadioet al. [2014]qui soutiennent qu' "A la suite de l'Initiative de Bamako en 1987, les politiques publiques sanitaires en Afrique ont privilégié le paiement direct. [Alors que] Dans sa formulation, l'Initiative de Bamako prévoyait que des mesures d'exemption soient prises pour ceux qui ne peuvent pas payer. Toutefois, la mise en œuvre de l'Initiative de Bamako a ignoré cette disposition. Par conséquent, son application dans les formations sanitaires a réduit l'accès aux soins de santé d'une grande partie de la population pauvre ».

Ces évasions ou fuites clandestines des malades hospitalisés avaient représenté une perte de 1858 USD (6,5\%) dans la mobilisation des recettes des hospitalisations des malades. Ces manques à gagner dans les finances de l'HGR de Manono avaient eu des conséquences sur la réalisation des objectifs retenus à être réaliser au courant des années 2019 et 2020. En effet, tout gestionnaire élabore le budget qui intègre les possibles sources des revenuesfinancières. Tout handicap en faveur de ces revenues aura donc des conséquences immédiates et à moyen terme dans la gestion institutionnelle : payement de personnel, achat des fournitures de bureau, achat des médicaments, achat des réactifs de laboratoire d'analyses biomédicales, etc. D'où le gestionnaire est appelé à empêcher la continuité des évasions des malades.

Les caractéristiques des malades évadés, dans notre contexte, plaident en faveur des conséquences de la pauvreté et du faible niveau d'instruction [Roquet 2011; Jean Pascalet al. 2006]. Cette pauvreté est accentuée par les conséquences des conflits intercommunautaires entre les peuples autochtones TWA et les populations Bantu locales, en majorité de l'ethnie LUBA (descendant de Ilunga Mbidi-A -Kiluwe). La population locale exerce les activités agricoles comme principale source de survie, alors que l'accès aux champs constitue un risque vital : les autochtones Twa (chasseurs) bloquant le passage et pillant les récoltes.

La santé du pauvre est préoccupante, surtout dans les pays en voie de développement. La pauvreté demeure un facteur essentiel à la non fréquentation des structures hospitalières, surtout celles de haute qualité, et favorise la mortalité [Larcher 2007 ; Mulinganyaet al. 2018].D'où la nécessité d'une attention particulière pour cette grande catégorie de la population.

Les auteurs des évasions sont des vrais génies. Durant nos investigations, il s'est avéré que plusieurs patients ou leurs membres de famille ciblent la nuit ou vers 5 heures du matin, lorsque les infirmiers dorment encore, pour s'évader. A cet effet, les malades sortent sans bagages (déjà expédiés la veille par les membres de la famille venues en visite) et après ce sont les femmes qui sortent avec leurs enfants au dos trompant la vigilance de la sentinelle. L'absence de clôture à l'HGR de Manono facilite ces opérations à cause de la multiplicité des portes d'entrée et de sortie. 
C'est pourquoi nous insistons sur le besoin de communication adaptée pour le changement des mentalités, les mesures incitatives au développement intégral des communautés locales et le système de tarification adapté à la pauvreté du milieu local.

\section{CONCLUSION}

L'évasion des malades, reflet de la pauvreté dans notre milieu, est une triste réalité qui impacte négativement sur la mobilisation et la maximisation des recettes dans les structures sanitaires : cas spécifique de Manono, dans le contexte de cette étude. L'évasion des malades doit être endiguée car plusieurs patients qui s'évadent reviennent plus tard avec des complications graves et en plus, l'administration de l'hôpital fait des déficits financiers énormes dans ses rapports de trésorerie : ce qui ne permet pas d'atteindre les objectifs annuels et satisfaire au payement de prime des prestations locales des agents.

Dans notre contexte de pauvreté, la solution aux évasions des malades passe par la lutte contre les inégalités socioéconomiques, la concertation et l'implication des autorités politiques, administratives et coutumières dans la gestion commune de l'hôpital, et la mobilisation des populations au changement de mentalité. Les gestionnaires des structures hospitalières doivent contribuer dans la construction des clôtures solides ayant une grande porte pour les véhicules et une petite porte pour les piétons, Installer un service de sécurité (sentinelle) permanent et vigilant avec mission de contrôle rigoureux sur les flux des entrées et sorties de l'hôpital, infligerdes sanctions disciplinaires à tout personnel soignant surpris dans la complicité d'évasion avec les malades, et instaurer un système d'allégement financier aux pauvres (indigents).

Conflit d'intérêt : Les auteurs ne déclarent aucun conflit d'intérêt en rapport avec cette étude. Cette étude a été financée par les contributions propres des auteurs.

Remerciements : Les auteurs remercient sincèrement les étudiants de l'Institut Supérieur des Techniques de Manono et les infirmiers (e) de l'Hôpital général de référence de Manono pour leur participation dans la récolte des données.

Contribution des auteurs : Tous les auteurs ont contribué dans la réalisation de cette étude. De manière plus particulière : (1) JLBL a participé à la conception, la supervision de la récolte des données et à la préparation du manuscrit ; (2) MKD a participé à la conception et la supervision de la récolte des données ; (3) HKB a contribué à l'analyse statistique des données, préparation du manuscrit, supervision de l'étude et à la lecture de la version finale ;(4) SKK à la récolte des données, à la préparation du manuscrit et à la lecture de la version finale ; (5) NSRà la récolte des données, à la préparation du manuscrit et à la lecture de la version finale

\section{REFERENCES}

[1] Chernichovsky D, Meesook OA (1986). Utilization of health services in Indonesia. SocSci Med.23(6):611-620. http://dx.doi.org/10.1016/0277-9536(86)90155-3

[2] Connolly Mark P., Nikolaos Kotsopoulos ,Postma Maarten J., Aomesh Bhatt (2017). The Fiscal Consequences Attributed to Changes in Morbidity and Mortality Linked to Investments in Health Care: A Government Perspective Analytic Framework. Value in health 20, 273 277 http://dx.doi.org/10.1016/j.jval.2016.11.018

[3] Jean Pascal, Hélène Abbey-Huguenin et Pierre Lombrail (2006). Inégalités sociales de santé : quels impacts sur l'accès aux soins de prévention? Lien social et Politiques-RIAC, 55, La santé au risque du social. Printemps, 115-124 DOI : https://doi.org/10.7202/013229ar

[4] KabindaMaotela J, Patrick MulopoMitashi, Faustin MukalengeChenge (2019). Analyse des modalités de financement des soins de santé en République démocratique du Congo : une revue systématique. Annales Africaines de Médecine, 12 (2) : e3203 - e3219

[5] Kadio K, Ridde V, Mallé Samb O (2014). Les difficultés d'accès aux soins de santé des indigents vivant dans des ménages non pauvres. Santé Publique, 26, 89-97. https://doi.org/10.3917/spub.137.0089

[6] Larcher P. (2007). Principales répercussions de la pauvreté sur la santé. Laennec, 55, 15-26. https://doi.org/10.3917/lae.074.0015 
DOI: $\underline{10.51386 / 25815946 / i j s m s-v 4 i 5 p 120}$

Volume: 4 Issue: 5

September to October 2021

https://www.ijsmsjournal.org

[7] ManzambiKuwekita J, ElokoEyaMatangelo G, Bruyère O, Gosset C, Guillaume M, Reginster JY (2015). Financement de la santé et recouvrement des coûts : le lourd fardeau des ménages congolais, Résultats des comptes nationaux de la sante. Journal d'Epidémiologie et de Santé Publique, (JESP), 15: 15-27

[8] MSP/PNCNS (2016). Rapport des comptes nationaux de la santé 2014. MSP. Kinshasa (Congo). 68p., 17-48

[9] Mulinganya V, Asima F, Mirindi P, Karemere H (2018). Temps d'attente prolongés aux services de consultation médicale : enjeux et perspectives pour des hôpitaux de Bukavu en République Démocratique du Congo. Pan African Medical Journal, $29: 173$. doi:10.11604/pamj.2018.29.173.13651 This article is available online at: http://www.panafrican-medjournal.com/content/article/29/173/full/

[10] Roquet D (2011). Bernard Kouassi, Pauvreté des ménages et accès aux soins de santé en Afrique de l'Ouest .Territoire en mouvement Revue de géographie et aménagement [En ligne], mis en ligne le 21 mars 2017, consulté le 21 octobre 2021. URL: http://journals.openedition.org/tem/1301; DOI : https://doi.org/10.4000/tem.1301 